\title{
Human Ehrlichioses in Brazil: First Suspect Cases
}

Simone B. Calic ${ }^{1}$, Márcio A.M.Galvão ${ }^{2,7}$, Fátima Bacellar ${ }^{6}$, Christiane M. B. M. Rocha ${ }^{3}$, Cláudio L. Mafra ${ }^{5}$, Romário C. Leite ${ }^{4}$ and David H. Walker ${ }^{7}$

\begin{abstract}
Ezequiel Dias Foundation ${ }^{1}$, Belo Horizonte; Department of Clinical and Social Nutrition ${ }^{2}$, Federal University of Ouro Preto, Ouro Preto; Federal University of Lavras ${ }^{3}$, Lavras; Federal University of Minas Gerais, School of Veterinary"; Department of Biochemistry and Molecular Biology ${ }^{5}$, Federal University of Viçosa, Viçosa, Minas Gerais, Brazil; National Institute of Health Dr. Ricardo Jorge ${ }^{6}$, Águas de Moura, Portugal; World Health Organization Collaborating Center for Tropical Diseases, University of Texas Medical Branch ${ }^{7}$, Galveston, USA.
\end{abstract}

\begin{abstract}
Brazilian spotted fever (BSF) rickettsiosis is the most common and recognized of the human rickettsioses in Brazil. It is difficult to establish the diagnosis of human rickettsiosis infection by routine microbiologic methods, creating a false idea that Rickettsia and Ehrlichia infections are rare and without importance. New tick-borne diseases, like Human Granulocytic Anaplasmosis (HGA) and Human Monocytic Ehrlichiosis (HME), have been described in many countries. These diseases can present symptoms similar to rickettsioses of the spotted fever group, and they are transmitted by ixodid ticks. The first two suspected cases of human ehrlichiosis in Brazil were first considered to be cases of BSF. The differential diagnosis was made at the Minas Gerais Rickettsiosis Public Health Laboratory. The clinical and laboratory findings, with positive serology for the HME agent, indicated suspected cases of human ehrlichioses in Brazil.

Key Words: Rickettsioses, human ehrlichioses, Brazilian spotted fever, Brazil.
\end{abstract}

Rickettsiae and Ehrlichiae were considered in the past as a form of life between viruses and bacteria. In truth they are Gram-negative bacteria that live inside the cell and do not grow out of the cell in their vectors or mammalian hosts [1,2]. With such a highly evolved niche, it is difficult to establish the diagnosis of human rickettsiosis infection by routine microbiologic methods, creating a false idea that Rickettsia and Ehrlichia infections are rare and without importance [3].

Brazilian spotted fever (BSF) rickettsiosis is the most common and recognized human rickettsiosis in Brazil. Recently, some of these bacteria previously considered

Received on 09 December 2003; revised 15 April 2004.

Address for correspondence: Dr. Simone Berger Calic. Rua Conde Pereira Carneiro, 80, Gameleira. Zip code: 30510-010, Belo Horizonte, Minas Gerais, Brazil. E-mail: sbcalic@funed.mg.gov.br

The Brazilian Journal of Infectious Diseases 2004;8(3):259-262 (C) 2004 by The Brazilian Journal of Infectious Diseases and Contexto Publishing. All rights reserved. as animal parasites, were recognized as pathogenic for humans, causing diseases such as human granulocytic anaplasmosis (HGA) and human monocytic ehrlichiosis (HME). These diseases can present symptoms similar to spotted fever rickettsiosis, and they are transmitted by ixodid ticks $[4,5]$.

The American medical literature describes patients with human ehrlichiosis who usually visit a physician's office during the first week of the disease subsequent to an incubation period of 5 to 10 days after the tick bite [6]. The symptoms include fever, headache and myalgia. Other signs and symptoms include nausea, vomiting, diarrhea, cough and mental confusion. In contrast to BSF, the presence of a rash is less common in HME patients. However, renal failure, intravascular coagulation disorders, meningoencephalitis, adult respiratory distress syndrome, and coma may occur in severe cases. Pleocytosis in cerebrospinal fluid is associated with human ehrlichioses [3,7-9]. These diseases have not been described before in humans in 
Brazil, and Ehrlichia has not been described in arthropod vectors from Brazil. Evidence of antibodies reactive to Ehrlichia chaffeensis was reported for the first time in 2002 in a serologic survey of dogs from a spotted fever endemic region in Minas Gerais state [10]. The implementation of specific techniques that permit distinguishing between the different rickettsioses has been fundamental to elucidate infections such as ehrlichioses and other rickettsial diseases.

\section{Materials and Methods}

The suspected cases were detected by an epidemiological surveillance system in Minas Gerais state as suspected cases of BSF, and the sera of these patients were examined by indirect immunofluorescence assay [11]: Ten microliters of diluted sera were added to each well of the antigen slides for $R$. rickettsii and E. chaffeensis, which were incubated for $30 \mathrm{~min}$ in a humid chamber. The antigen used was obtained from the PANBIO Laboratory. The slides were rinsed once and washed in PBS ( $\mathrm{pH} 7.1$ ) for 10 minutes, twice. Fluorescein isothiocyanate-conjugated goat anti-human $\operatorname{IgA}, \operatorname{IgG}$ and $\operatorname{IgM}(\mathrm{H}+\mathrm{L})$ (Biolab Laboratories) was used at the optimal working dilution of $1 / 100$ as the secondary antibody. After incubation for $30 \mathrm{~min}$., the slides were rinsed once in PBS for 10 minutes, then washed in PBS ( $\mathrm{pH} 7.1$ ) containing Evans blue for 5 minutes, and then in sterile water for 5 more minutes. The slides were mounted with $90 \%$ glycerin in PBS under coverslips and observed under epifluorescence with an ultraviolet microscope (Zeiss, M C 80 DX) at 40X magnification. End point titers were determined by examination of serial two-fold dilutions of the reactive sera.

\section{Results and Discussion}

Case 1

A 39 year old man from Monte Carmelo Municipality, Minas Gerais state, Brazil was suspected to have BSF. The outset of the symptoms occurred on May 8, 2001, with fever, headache, nausea, vomiting, myalgia, conjunctivitis, respiratory insufficiency and renal failure. The patient had a clinical history of immunosuppression, pneumonia and chronic renal failure. He had been exposed to a dog that hunted rats.

Laboratory results on May 28, 2001: 1,600/ $\mathrm{mm}^{3}$ white blood cells; $2,800,000 / \mathrm{mm}^{3}$ red blood cells; $8.8 \mathrm{~g} / \mathrm{dL}$ hemoglobin; $25.8 \%$ hematocrit; and 42,000/ $\mathrm{mm}^{3}$ platelets. The IFA serologic reactions for BSF and murine typhus, macroagglutination test for leptospirosis, and ELISA for yellow fever and dengue revealed no antibodies. The presence of HME and HGA antibodies was determined by IFA, with the following results: IgM 1/64, IgG 1/128 and antihuman $\operatorname{Ig} \mathrm{A}, \operatorname{IgG}$ and $\operatorname{IgM}(\mathrm{H}+\mathrm{L}) 1 / 512$ to Ehrlichia chaffeensis. HGA antibodies were not detected.

Antibiotic therapy with chloramphenicol was initiated on May 29, with a good clinical response observed at 48 hours. A second sample of serum was not collected.

\section{Case 2}

A 20-year-old man lived in Ponte Nova Municipality, Minas Gerais State, Brazil. On May 15, 2001, he noted the onset of fever, headache, myalgia, conjunctivitis, nausea and vomiting. He developed icterus, respiratory insufficiency, renal failure, diarrhea, hepatomegaly, malnutrition and mental confusion by May 20. Laboratory results on May 20, 2001, revealed pleocytosis of the CSF, 240 IU/L oxalacetic transaminase, $53 \mathrm{IU} / \mathrm{L}$ pyruvic transaminase, $13.85 \mathrm{mg} /$ $\mathrm{dL}$ direct bilirubin, and $22.61 \mathrm{mg} / \mathrm{dL}$ indirect bilirubin . The serologic reactions for BSF and murine typhus by IFA, leptospirosis by macroagglutination test, and yellow fever and dengue by ELISA revealed no antibodies; but antibodies against $E$. chaffeensis (IgM 1/64, IgG 1/256 and anti-human IgA, IgG and IgM $(\mathrm{H}+\mathrm{L}) 1 / 64)$ were detected, with no reaction to HGA. The patient died on May 25, 2001.

These diagnoses of HME were made based on clinical and serologic results. The authors agree that hepatic enzyme changes in patient 2 are due to acute 
Figure 1.

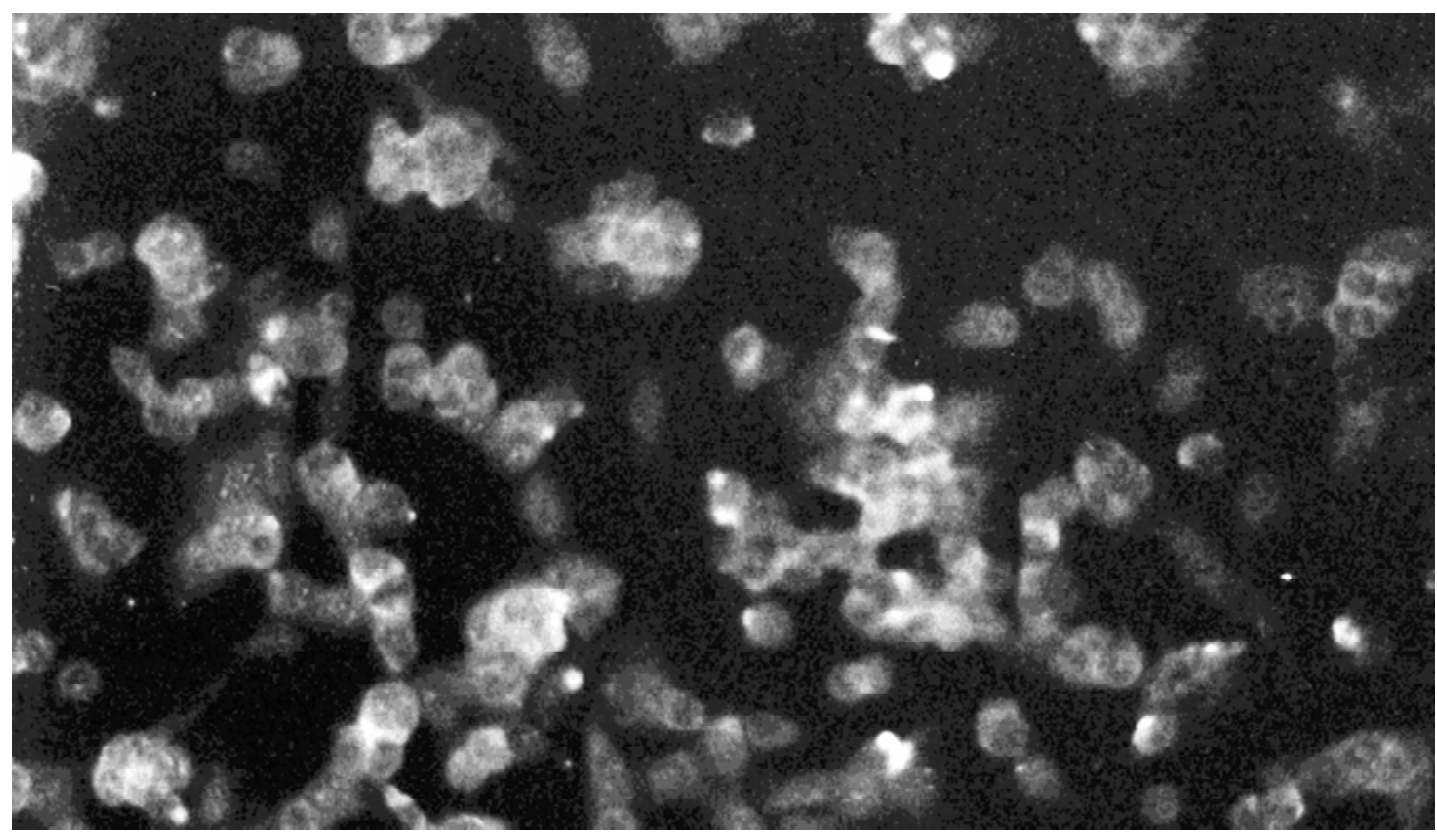

Figure 2.

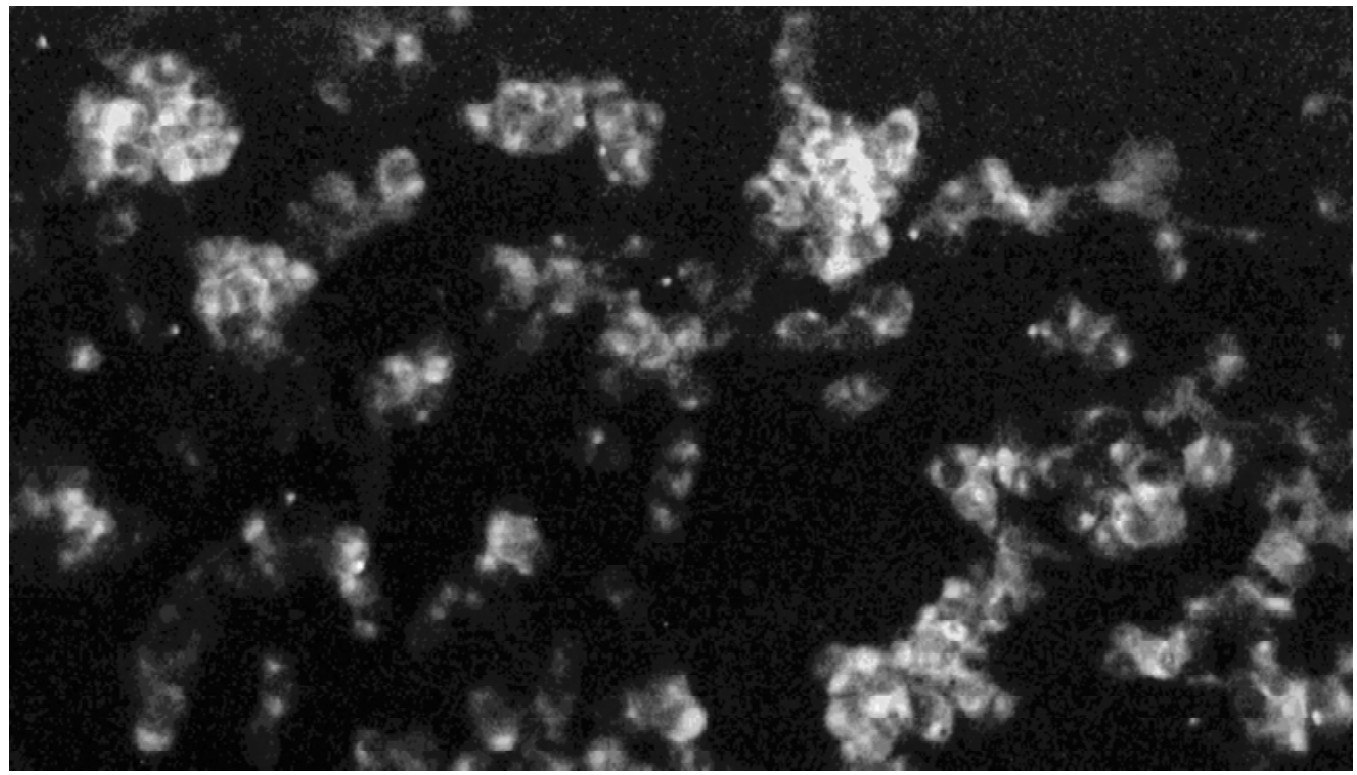


illness. This patient had no history of previous hepatic or hematological diseases, and the possibility of hepatic renal syndrome being responsible for hepatic enzyme changes was also discarded based on clinical findings. The peripheral smears for morula were not reexamined because the physicians were not thinking of this diagnosis when the cases occurred. The serology does not confirm a diagnosis, but it can be suggestive. In our cases, the strong fluorescence by IFA to E. chaffeensis, the negative results for other rickettsioses and the significant titers of antibodies obtained for $\operatorname{IgM}$ and $\operatorname{IgG}$ to E. chaffeensis collaborate to place these cases in the category of suspected cases.

These first reported suspected cases of human ehrlichioses in Minas Gerais State, Brazil, show us the necessity of further studying the epidemiology of vectors and agents involved in this pathology, as well as in other rickettsioses. The finding of these agents in the environment increases the effectiveness of the diagnosis. Also, the correct diagnosis is important to initiate early treatment to significantly reduce the case fatality-ratio. Moreover, this could be important in differential diagnoses of hemorrhagic fevers.

\section{References}

1. Anderson B.E., Dawson J.E., Jones D.C., Wilson K.H. Ehrlichia chaffeensis, a new species associated with human ehrlichiosis. Journal of Clinical Microbiology 1991;29(12):2838-42.

2. Wen B., Rikihisa Y., Mott J., et al. Ehrlichia muris sp. nov., identified on the basis of $16 \mathrm{~S}$ rRNA base sequences and serological, morphological, and biological characteristics. International Journal of Systematic Bacteriology 1995;45:250-4.

3. Walker D.H., Dumler J.S. Emergence of ehrlichioses as human health problems. Emerging Infectious Diseases 1996;2(1):18-29.

4. Eng T.R., Harkess J.R., Fishbein D.B., et al. Epidemiologic, clinical, and laboratory findings of human ehrlichiosis in the United States, 1988. Journal of the American Medical Association 1990;264:251-8.

5. Jacobs R.F. Human monocytic ehrlichiosis: similar to Rocky Mountain spotted fever but different. Pediatric Annals 2002;31(3):180-4.
7. Walker D.H., Dumler J.S. Human monocytic and granulocytic ehrlichioses. Discovery and diagnosis of emerging tick-borne infections and the critical role of the pathologist. Archives of Pathology Laboratory Medicine 1997;121(8):785-91.

8. Devereaux C.E. Human monocytic ehrlichiosis presenting as febrile diarrhea. Journal of Clinical Gastroenterology 1997;25(3):544-5.

9. Ratnasamy N., Everett E.D., Roland W.E., et al. Central nervous system manifestations of human ehrlichiosis. Clinical Infectious Diseases 1996;23:314-9.

10. Galvão M.A.M., Lamounier J.A. , Bonomo E., et al. Emerging and reemerging rickettsiosis in an endemic area of Minas Gerais State, Brazil. Cadernos de Saúde Pública 2002;18(6):1593-7.

11. Centers for Diseases Control and Prevention. Division of Viral and Rickettsial Diseases: Indirect fluorescent antibody technique for the detection of rickettsial antibodies. Atlanta: National Center for Infectious Diseases, s.d;11, 2001. 